\title{
CONCEPCIONES SOBRE CUERPO EN LA FORMACIÓN DE MAESTROS DE EDUCACIÓN FÍSICA DE LA UNIVERSIDAD DE LOS LLANOS
}

\author{
BODY CONCEPTIONS IN PHYSICAL EDUCATION TEACHER TRAINING AT UNIVERSIDAD DE \\ LOS LLANOS
}

Fernando Campo Polo ${ }^{1}$

\section{Resumen}

\begin{abstract}
El estudio aquí presentado buscó comprender las concepciones de cuerpo que subyacen en la formación de maestros del programa de Licenciatura en Educación Física y Deportes de la Universidad de los Llanos, y cómo estos se develaron en el currículo. Para dicho análisis, se acudió a la aplicación de una metodología que permitiera vislumbrar la tendencia y la agenda investigativa de docentes, para prospectar con objetividad dicha situación. Se debió, entonces, abordar la metodología del rastreo arqueológico a partir de la descripción de las condiciones conceptuales del saber objeto de estudio. Los resultados se antojan inquietantes e interesantes, en tanto revelación documental, partiendo de ciertas categorías de análisis que dan cuenta del trasegar del cuerpo en el currículo a partir de 1998. Finalmente, se destaca el estado de la presente investigación, en tanto resultados parciales, debidos, fundamentalmente, a la revisión archivística y documental que en materia de normas, políticas y directrices institucionales develan prácticas discursivas sobre el cuerpo, lo que posibilita una primera aproximación e interpretación.
\end{abstract}

Palabras clave: cuerpo y educación física, currículo y educación física, formación de maestros

\section{Abstract}

This text sought to understand the conceptions about body underlying teacher training in the program Degree in Physical Education and Sports at Universidad de los Llanos, and how these were unveiled in the curriculum. For this analysis, we came to the application of a methodology that gave a glimpse of the trend and the research agenda of teachers, to prospect the situation objectively. It must then deal with the methodology of archaeological tracking from the description of the conceptual conditions of knowledge under study. The results seem disturbing and interesting, as documentary disclosure, based on certain categories of analysis showing the path of the body in the curriculum since 1998. Finally, the state of this research is highlighted, as partial results, due mainly to the archival and documentary revision that in standards, policies and institutional guidelines reveal discursive practices on the body, which allows a first approach and interpretation.

Keywords: body and physical education, physical education and curriculum, teacher training

Fecha de recepción: 4 de diciembre de 2015

Fecha de aprobación: 4 de mayo de 2016

Para citar este artículo:

Campo, P. (2016). Concepciones sobre cuerpo en la formación de maestros de educación física de la Universidad de los Llanos. Lúdica Pedagógica,24,69-78.

$1 \operatorname{Dr}(\mathrm{c})$ en Educación. Magíster en Administración pública. Licenciado en Educación Física. Profesor titular de la Universidad de los Llanos. Correo electrónico: campospolofernando@gmail.com 


\section{INTRODUCCIÓN}

La sociedad contemporánea, y en especial la colombiana, tiene enormes esperanzas en que la educación se convierta en la catapulta que posibilite salir de la encrucijada social en la que se encuentra el país. El sistema educativo tiene como objetivos, entre otros, transformar la realidad y alcanzar niveles óptimos de equidad social. Debe ser perentorio para todo programa de formación en educación superior y, por supuesto, para las expectativas sociales y del Estado, estar orientados hacia la educación de calidad, como estrategias de inclusión y movilidad social. Es en este sentido en el que se demanda de un programa universitario en formación de maestros que garantice la reflexión constante de su quehacer desde la intencionalidad permanente de su propuesta curricular.

Se pretendió, entonces, para el caso de la educación física de la Universidad de los Llanos, interpretar sus propósitos formativos a lo largo de su historia como programa formador de docentes, y compararlos con los actuales, y con ello, descifrar los esquemas teóricos sobre los que se forjan los programas curriculares, los cuales, a su vez, deberán denotar las características más simbólicas de quienes intervinieron en su formulación. Se destacan, sin lugar a dudas, los intereses de los diferentes grupos de poder en el programa académico, así como sus tendencias teóricas, de las cuales se reconocerán, seguramente, distintas corrientes en los programas curriculares.

La importancia del presente estudio radicó en la claridad particular de los niveles de intencionalidad formativa, lo cual permitió bosquejar el derrotero por el que quiere transitar cada programa, y permitió así inferir cuáles son las tendencias más marcadas en la formación de licenciados en educación física para el caso de la Universidad de los Llanos y cuál es su intención futura, lo cual no ha sido indagado con suficiencia hasta el día de hoy.

Finalmente, cabe destacar que el programa de Licenciatura en Educación Física y Deportes de la Universidad de los Llanos ha mostrado en su acontecer curricular una tendencia marcada hacia la pedagogía y la investigación, las cuales aparecen en la malla curricular como ejes transversales, pero muestran también un distanciamiento respecto a la retórica tradicionalmente deportivista y eficientista del cuerpo. Un análisis que, a priori, plantea la necesidad de hacer efectivo un riguroso estudio, desde un enfoque metodológico relativamente innovador, como lo es el arqueológico-genealógico, por demás interesante, en el campo profesional de la educación física.

\section{METODOLOGÍA PROPUESTA}

La arqueología, como proceso investigativo, indagó con profundidad el análisis de los enunciados, y permitió así describir los contextos a los que pertenecen y las condiciones dadas en un saber específico. Para el caso de la Educación Física, se concibió el reconocimiento histórico del cuerpo, visibilizado desde los enunciados. Dicho análisis epistémico, dado por la arqueología, conllevó determinar cuáles fueron los discursos consolidados y su relación con las prácticas educativas, y cómo estas adquieren un significado en la convergencia de las prácticas sociales. La arqueología, como caja de herramientas, coincidió con la intencionalidad de la propuesta investigativa, desde donde se intentó inferir la capacidad discursiva del maestro en educación física dentro del marco de la heterogeneidad de los saberes implícitos en el currículo. Así las cosas, la arqueología permitió una interrogación acerca de cuáles fueron los procedimientos del cuerpo en la educación física, a qué leyes obedecieron y cómo se decantaba en el discurso disciplinar la formación de enunciados. Por otra parte, se establecieron su coherencia y su función dominante y, por supuesto, se verificó su importancia en la formación profesional de maestros en educación física. La arqueología determinó así los niveles del saber y cómo estos se correlacionan con las prácticas.

Se vislumbró el análisis propio de la genealogía estableciendo lo relativo al poder y sus relaciones. Se visualizó, también, la procedencia del discurso del cuerpo y cómo este se da en la interpretación del docente y en el proceso de formación que el docente ha recibido. Dicho análisis genealógico permitió detectar cómo el supuesto de verdad surge en el concepto, y cómo este, a su vez, se interrelaciona con el discurso y posteriormente adquiere vigencia a través de la práctica.

El enfoque genealógico permitió hacer una profunda búsqueda en torno al discurso sobre cuerpo a partir del cual ha transitado la formación de maestros de educación física en la Universidad de los Llanos; ello permitió una interpretación disciplinar y estableció circunstancias y dispositivos en una pretendida cientificidad. Estos agenciamientos determinaron desde la 
genealogía cómo la maquinaria propia del currículo se transpola en la sociedad y cómo un campo de estudio como el de la educación física se interrelaciona con las prácticas discursivas sobre cuerpo, a partir del análisis genealógico, desde los acontecimientos académicos y cotidianos de la vida universitaria. Así pues, uno de los objetivos del análisis genealógico fue evaluar la funcionalidad del poder detectando su mecánica y explicando cómo el discurso del maestro se convierte en un dispositivo de múltiples intereses, a partir de la implementación de técnicas, tácticas y estrategias para interrelacionar el saber con el poder.

La tendencia investigativa, arqueológica y genealógica desde la teoría foucaultiana implicó el uso de instrumentos para recolectar información tales como la entrevista en profundidad, la cual, además de ser propia de la investigación social cualitativa, presenta como característica el hecho de ser abierta y emergente, por lo que facilita una verdadera exploración, con una focalización plena y una profundización puntual, todo lo cual permite una unidad de análisis gracias a la que se pueden plantear presupuestos teóricos los cuales, además, admitirán el enfoque de nuevos procedimientos. La pertinencia de dicho instrumento radicó en la “(...) comprensión del campo social que rodea al individuo, yendo más allá de su historia de vida en sí misma" (Fideli \& Marradi, 1996, p. 195). Lo que interesa a este respecto es mostrar un panorama de engranajes entre el sujeto y su entorno sociohistórico.

Adicionalmente, se incluyó entre los instrumentos para recolectar información la revisión documental; se acudió, ante todo, a la revisión bibliográfica, a la revisión de normas, leyes, decretos, resoluciones, planes curriculares, etc. A este respecto, se entendió la revisión documental como el proceso dinámico por el cual se recogió, clasificó, recuperó y analizó la información. Y aunque a escala operativa el proceso de revisión documental se desarrolló de forma independiente del enfoque metodológico utilizado, "no ocurrió lo mismo con su uso, sentido y presentación” (Latorre, Rincón \& Arnal, 2003, p. 58). Esto es, se intentó utilizar dicha información en función de la comprensión y el análisis del objeto de interés de la propuesta en general.

\section{HALLAZGOS}

En procura de definir mecanismos que posibilitaran el análisis sobre prácticas discursivas en torno al cuerpo en la formación de maestros, se hizo necesario plantear una serie de herramientas y métodos que dieran cuenta de búsquedas, selecciones y debidos tratamientos a los archivos documentales, en la medida en que ello permitiera interpretaciones a la luz del enfoque foucaultiano ${ }^{2}$. Para ello, se acudió a dos rutas de análisis: localización de fuentes documentales y lectura de registros.

Respecto a la primera, se ubicaron el inventario documental y los archivos por consultar, así como las instituciones en las que reposaran dichos documentos, y la clasificación de estos. Dicha localización buscó dar cuenta del tránsito curricular en tiempos disímiles; esto es, un discurrir temporal sobre el antes y el después del Decreto 272 de 1998, entendido como el que genera cierta ruptura en la formación de maestros y la concepción sobre cuerpo, pues su entrada en rigor genera efectos y virajes conceptuales, y se establece, con ello, lo que se conoce como el núcleo del saber pedagógico, conformado por la educabilidad del ser humano, la enseñabilidad de las disciplinas y los saberes dentro de sus dimensiones histórica, epistemológica, social y cultural.

La localización, como primera fase en el desarrollo de esta ruta metodológica, da cuenta, como decíamos, de un tránsito curricular. Ello indica, adicionalmente, que hallar estos documentos permitió el análisis de periodos cruciales en dicha transición. Por un lado, el documento a., con el que se crea el programa en sus más primigenios inicios, seguido del documento b., el cual presenta - justificando al primero- ante los órganos y las dependencias de todo el país sus argumentos en torno a la necesidad de funcionamiento como programa de educación superior, en correspondencia con el Decreto Nacional 445 de 1981, Literal F, que plantea el "ofrecimiento a la comunidad beneficios de formación integral, capacitación, para responder a las necesidades regionales (...)". No obstante, pese a los esfuerzos por dar apertura al programa, este no logra rendir frutos, y finalmente es clausurado. Las razones para ello son,

2 Para ello, se recurre, en alguna medida, al planteamiento metodológico del Grupo de Historia de las Prácticas Pedagógicas en Colombia, y del Grupo del Doctorado Interinstitucional en Educación; específicamente, a partir de la propuesta metodológica sugerida por Martínez y Quiceno en el proyecto "Modernización, Subjetividad y Sociedad de Control en la educación en Colombia 1950-2000". 
de alguna manera, confusas, por lo que en este punto se fija la necesidad de acudir, eventualmente, al relato de actores que por entonces fungieron como protagonistas. Posteriormente, en el documento c., se reabre el programa con un actualizado enfoque curricular; sin embargo, se carece de información y de evidencia documental frente a ese importante periodo, por lo que también se sugiere acopio de información a través de entrevistas en profundidad a los agentes que, directa o indirectamente, se relacionaron con dicho momento.
Seguidamente, el documento d. brinda información sobre otro importante periodo curricular, a partir del que se infieren prácticas discursivas sobre el cuerpo que son de especial interés para el análisis propiamente dicho. Finalmente, se tiene el documento e., que representa la actual y más vigente propuesta en torno a la formación de maestros, y que, seguramente, arrojará pistas en lecturas o documentación que profundice con mayor rigor el levantamiento del archivo.

Tabla 1. Inventario archivístico sobre normativa y documentación institucional.

\begin{tabular}{|l|l|l|l|}
\hline \multicolumn{1}{|c|}{ Documento } & \multicolumn{1}{|c|}{ Fuente documental } & \multicolumn{1}{c|}{ Institución (ubicación) } & \multicolumn{1}{c|}{$\begin{array}{c}\text { Clasificación } \\
\text { documental }\end{array}$} \\
\hline a. & $\begin{array}{l}\text { Acuerdo 038 de 1982, por el cual se } \\
\text { aprueba la apertura del Programa en } \\
\text { Educación Física y Deportes. }\end{array}$ & $\begin{array}{l}\text { Universidad de los Llanos, Oficina de } \\
\text { Archivo y Correspondencia. }\end{array}$ & Normativo \\
\hline b. & $\begin{array}{l}\text { Informe presentado al ICFES para soli- } \\
\text { citud de Licencia de Funcionamiento, } \\
\text { Volumen I, 1983. }\end{array}$ & $\begin{array}{l}\text { Universidad de los Llanos, Oficina de } \\
\text { Planeación. }\end{array}$ & Normativo \\
\hline c. & $\begin{array}{l}\text { Acuerdo 120 de 1993, por el cual se } \\
\text { crea el programa de Licenciatura en } \\
\text { Educación Física y Deportes. }\end{array}$ & $\begin{array}{l}\text { Universidad de los Llanos, Oficina de } \\
\text { Archivo y Correspondencia. }\end{array}$ & Normativo \\
\hline Decreto 272 de 1998 & $\begin{array}{l}\text { Una propuesta curricular para el nuevo } \\
\text { milenio. ¿Cómo formar un profesional } \\
\text { de la educación física? Primera edición, } \\
\text { 2000. }\end{array}$ & $\begin{array}{l}\text { Universidad de los Llanos, Biblioteca; } \\
\text { Instituto Municipal de Deporte y } \\
\text { Recreación. }\end{array}$ & Texto de reflexión \\
\hline d. & $\begin{array}{l}\text { Acuerdo 007 de 2002, por el cual se } \\
\text { establecen las políticas y los lineamien- } \\
\text { tos curriculares de la Universidad de } \\
\text { los Llanos. }\end{array}$ & $\begin{array}{l}\text { Universidad de los Llanos, Oficina de } \\
\text { Archivo y Correspondencia }\end{array}$ & Normativo \\
\hline e. &
\end{tabular}

Por otro lado, en lo que tiene que ver con la lectura de los registros, se pretendió, de manera fundamental, la exploración de fuentes documentales con el fin de contar con criterios para seleccionar los que demandan una lectura en profundidad. Para ello, se hace uso de una matriz de análisis identificando los diferentes momentos curriculares representados en los documentos de la primera fase (a, b, c y e). Dicha matriz, entonces, establece como criterios de análisis: definición, enfoque curricular, propósitos de formación y perfil profesional y ocupacional. Con ello, estableceremos aquí, de manera suficientemente clara, cuáles son las convergencias y las divergencias a la luz de la interpretación efectuada, en tanto se subrayan los apartados que reflejan la importancia para cada uno de los momentos y los criterios.

Una primera lectura en torno a definiciones, relaciones y predominancia conceptual a lo largo de la historia curricular del programa de Licenciatura en Educación Física y Deportes, da cuenta, en principio, de un alto contenido técnico y tecnológico en tanto destrezas y habilidades docentes, desde el entendido de la instrumentalización del conocimiento reflejado en los procesos de enseñanza-aprendizaje, y haciendo transición para un abordaje, en lo sucesivo, hacia principios de formación interdisciplinares, cuyos elementos como la crítica reflexiva y la vocación, se constituyan en aspectos que orienten el acceso al conocimiento a través de núcleos del saber pedagógico. Tal instancia permanece en el tercer documento curricular, y hace énfasis en competencias que posibiliten la interpretación y la transformación de la realidad social y cultural del entorno.

El análisis que deja el enfoque curricular en la transición de estructuras da cuenta, en principio, de un sustento en las eventualidades del contexto nacional e internacional en relación con los propósitos del sistema nacional de educación que propende por las competencias y las necesidades regionales. Así, también, se procura la preservación y el mejoramiento de la salud, como 
una salida a los influjos propios de la modernidad. Seguidamente, el énfasis propuesto plantea miradas contextualizadas como una alternativa de nuevas actitudes hacia la sociedad, el conocimiento y la cultura, a partir del estímulo de capacidades investigativas con la perspectiva de un currículo integrado capaz de generar sensibilidades educativas. Finalmente, es entendido de manera fragmentaria, a partir de elementos discursivos que propicien la acción y el sentido, en el despliegue de normas y articulaciones formales de un sujeto que permanentemente vive en interrelación con otros. Aquí la interdisciplinariedad funge como dinamizadora en la compresión del movimiento y el cuerpo, así como de las dimensiones que le son constituyentes. Se nombra con insistencia el desarrollo humano como un indicador de prevalencia en los procesos educativos del enfoque curricular propuesto, de cara al desenvolvimiento profesional en una eventual ciudadanía, que, desde una nueva visión reflexionada en torno a él y el mundo que lo rodea, propenda por una nueva conciencia, con nuevos valores como persona.

En lo que tiene que ver con propósitos de formación, dicho análisis refleja una constante preocupación por el perfeccionamiento y la actualización de los conocimientos, acorde ello con la realidad colombiana, a partir de cursos, seminarios, etc. Se opta, en el mismo sentido, por alternativas siempre encaminadas a la especialización de los saberes. Por otro lado, se evidencian preocupaciones por lo investigativo como una posibilidad que brinde habilidades para la búsqueda de la verdad, sin desatender la sensibilidad social que ello, necesariamente, implica. De forma paralela, se intenta, en la línea del saber pedagógico, trabajar por la formación de un sujeto capaz de reconocerse (y de reconocer a los otros) en el mundo con capacidad creativa y comprometido con la sociedad.

Finalmente, en cuanto a perfil profesional y ocupacional, se evidencia, desde el planteamiento curricular primario, una preocupación por lo científico y lo pedagógico, asumiendo su labor y su profesión como posibilidades para mejorar la calidad de vida de la sociedad. Se propende por un fomento de actitudes críticas e innovadoras, muy a pesar de que se sostienen intereses de aspecto, orientados a partir de la salud, la condición física y la presentación personal. Por otro lado, se evidencian búsquedas incesantes de la verdad a través de la investigación, como alternativa para la ampliación de los saberes desde una posición crítica. Se insiste en la necesidad de que el profesional y licenciado en educación física se esmere por la configuración de una actitud ética ante la sociedad, de modo tal que ello se refleje en la relación con los otros. El enfoque del segundo documento apunta también al desarrollo humano y al movimiento cargado de sentido en tanto subjetivo. Ello induce a una responsabilidad y una sensibilidad permanentes, que propicien elementos para el mejoramiento de la calidad de vida y el bienestar social. La interdisciplinariedad se constituirá, entonces, en un obligatorio pilar para el desarrollo de las actividades propias del educador, sin que esto implique un hacer por hacer.

Como parte del trabajo empírico, fungió como esencial la elaboración de un parangón que diera cuenta de las políticas oficiales en los planos nacional e internacional, y desde las cuales se expresaran las relaciones conceptuales y epistemológicas propias del campo profesional de la educación física, la recreación y el deporte. Adicionalmente, dicho trabajo permitió associar una serie de articulaciones e ideas en torno al cuerpo (corporalidad o corporeidad, según sea el caso), y así entender cómo estas, de alguna forma, generaron o no efectos en el ámbito institucional, sobre las prácticas discursivas en la formación de maestros del programa Licenciatura en Educación Física y Deportes de la Universidad de los Llanos.

Cada una de las políticas que a continuación se expondrán, además de referir nociones generales y específicas sobre su fundamentación y su implementación en el sistema educativo formal o no formal del país, guardan relación discursiva con conceptos en torno al cuerpo, contenidos en los campos disciplinares previamente mencionados; a saber: educación física, recreación o deporte. Por ello, se hace importante generar un análisis descriptivo de cada uno de los rasgos sobre el cuerpo, considerados y hallados en los documentos que, a propósito, se presentan como los de mayor relevancia, debido a su impacto y acogida en las instituciones dedicadas a la enseñanza y a la práctica de la educación física, como la nuestra.

El documento titulado Lineamientos Curriculares de la Educación Física trae a colación el concepto de dimensión corporal para dar cuenta de las posibilidades del cuerpo. Expone entre líneas que la dimensión corporal 
ha sido abordada por distintas disciplinas (Pedraza, 1998), que han producido un conjunto de conocimientos para explicar, desde distintas miradas, los problemas y los fenómenos que rodean la existencia corpórea del ser humano. Por un lado, la medicina ha producido un conjunto de saberes sobre prevención, rehabilitación y cuidado en busca de la salud, la prolongación de la vida y el bienestar. Por otro, la sociología ha explorado teorías sobre el cuerpo como objeto de control de consumo y como terreno de emancipación, tal como se menciona en los Lineamientos curriculares de la Educación Física. La psicología y la biología han explorado el esquema corporal, la imagen corporal, el talento corporal, el sentido cinestésico y otros puntos de vista antropológicos, filosóficos e históricos que centran su atención en la expresión, la experiencia corporal, la percepción, la sensibilidad y la comunicación.

Abordar la dimensión corporal implica tener en cuenta que existe una relación entre cuerpo y sociedad. Por un lado, la percepción del cuerpo depende de la construcción que la sociedad haya hecho de él, y, por otro, reconocer que en el cuerpo se manifiesta una determinada concepción de sociedad. Si, como lo plantea Mary Douglas (citada en Mockus, 1992), el cuerpo es una metáfora de la sociedad a través del cual se expresan juegos, ritos, mitos, prácticas y deportes, es posible hallar en el conocimiento del cuerpo no solo una explicación de lo que somos, sino también, claves inesperadas de lo que debemos ser; ello, como un análisis de lo que refleja el documento.

Estas relaciones se establecen a través de los usos sociales, de las prácticas y las técnicas del cuerpo y de sus implicaciones en conceptos, ideas, símbolos y principios éticos y morales. Las formas de vivir, de comer, de desplazarse, de trabajar, de divertirse o de atender la enfermedad llevan a usos y representaciones del cuerpo que en las sociedades contemporáneas lo disponen como objeto de destrucción o de liberación. En otras manifestaciones, el cuerpo es objeto de construcción estética, signo de distinción, medio de rendimiento o espacio de espiritualidad. En el ser y el tener cuerpo se manifiestan las contradicciones de la sociedad.

Con base en lo expuesto y lo dicho por los lineamientos, desde el punto de vista de la educación física la dimensión corporal comprende la experiencia, las significaciones y los conceptos de la realidad corporal del ser humano, a partir de las acciones motrices y sus relaciones con diversos aspectos individuales y colectivos de la existencia.

El desarrollo de la dimensión corporal está relacionado con las demás dimensiones del desarrollo humano: cognitivas, comunicativas, éticas, estéticas, valorativas, que se incluyen en el currículo para atender diferentes perspectivas del ser humano hacia sí mismo y en su entorno social y físico, y generar prácticas de adaptación, formación y recreación. A través de ellas se apoyan el crecimiento y el desarrollo motor, el cuidado de sí mismo, el de los demás y el del entorno, el aprendizaje de habilidades y destrezas motrices y de interacción social, la interrelación con la naturaleza y la cultura, la construcción de lenguajes corporales, la adecuación de espacios de ambientes y la utilización del tiempo.

Seguidamente, se presenta desde el documento sobre Orientaciones Pedagógicas para la Educación Física, Recreación y Deporte una concepción formativa de lo corporal como la posibilidad de ser, estar y actuar en el mundo. Las explicaciones sobre el cuerpo giran en torno a la promoción del cultivo personal desde la realidad corporal del ser humano. De esta manera, lo que pretende la educación física deberá ser formar a estudiantes conscientes de su corporeidad ${ }^{3}$, que pueden alcanzar mejores condiciones de vida a través del cuidado de su salud y de una ética corporal basada en la comprensión de sí mismos y de su interacción con los otros, lo que les permitirá sostener relaciones armónicas con su entorno natural y social. Esto exige la formación de actitudes y modos de actuar que otorguen sentido a las prácticas, las técnicas, los saberes y los hábitos de actividad motriz, al hacer énfasis en acciones preventivas y participativas que fomenten la cooperación y la solidaridad. Por ello, son propósitos fundamentales de la enseñanza de la educación física el respeto por los ritmos vitales, la sana alimentación, los hábitos de higiene, el cuidado del medio ambiente y el de los espacios públicos.

Por otro lado, y desde el mismo documento, se menciona la competencia expresiva corporal, como la que debe entenderse dentro del marco de un conjunto de conocimientos acerca de sí mismo (ideas, sentimientos, emociones), de técnicas para canalizar la emocionalidad (liberar tensiones, superar miedos, aceptar su cuerpo),

3 Corporeidad: término introducido por el filósofo francés Merleau-Ponty para superar la noción del cuerpo como objeto. 
de disponibilidad corporal y comunicativa con los otros, a través de la expresión y la representación posible, flexible, eficiente y creativa, de gestos, posturas, espacio, tiempo e intensidades. Así mismo, se resaltan las técnicas del cuerpo y el movimiento como un concepto propio del campo cultural. Una noción retomada de Mauss, quien la entiende como "todo acto tradicional eficaz para el desenvolvimiento en la vida, determinado por las características de la sociedad y sus formas de desarrollo" (1979, p. 342). Con esta perspectiva, las técnicas del cuerpo no se limitan a los procedimientos calificados que llevan a la obtención de la destreza, sino que se incorporan a la vida cotidiana y a la propia evolución de la persona en sus aspectos lingüístico y cognitivo.

Para cumplir las exigencias del movimiento corporal del ser humano se producen prácticas, materiales, campos de juego y espacios públicos, y se transforma el paisaje con construcciones especializadas que forman parte de la cultura contemporánea, y así se atienden los modos o los estilos de vida de las personas. Las prácticas y las técnicas corporales son producto de la experiencia o la indagación, y su finalidad se constituye como patrimonio cultural, objeto de aprendizaje para el bienestar de los seres humanos. De este modo, las manifestaciones corporales y motrices representan un auténtico fenómeno social, conforman prácticas y técnicas del cuerpo y del movimiento, que, a la vez que consolidan el desarrollo del ser humano, se convierten en objetos de aprendizaje.

Por otro lado, el documento Plan Nacional de Educación Física, 2002-2006, que expone genéricamente las tendencias de la educación física, resume, simultáneamente, tres pilares de carácter universal para ella, a saber: el cuerpo, el juego y el movimiento, los cuales no solo constituyen la posibilidad de toda práctica de la cultura física, sino que también son su fuente de sentido y de significación, en tanto median toda relación del hombre con el mundo.

De acuerdo con lo anterior, el plan recoge en un todo armónico diferentes campos de actividad humana, en los cuales la educación física cumple distintas funciones, que realiza de manera independiente o interdisciplinaria, como la educación, la cultura ciudadana, la salud, la expresión corporal, la relación con el medio ambiente y el aprovechamiento del tiempo libre.
Por ello, se dice que el Plan Nacional de Educación Física, es un medio para orientar la intencionalidad y organizar las acciones necesarias con el fin de que la disciplina cumpla su objetivo de integrar el conocimiento y la construcción social en las actuales condiciones históricas, desde las cuales se intenta superar visiones reduccionistas propias del campo y la ciencia clásica. En efecto, no es posible que la educación física logre su propio desarrollo si no se articula a las necesidades del contexto social, para lo cual dispone, como su principal medio, del avance de un conocimiento teórico, técnico, pedagógico y organizativo que debe difundir, perfeccionar y diversificar a través de su extensión cultural y social, en vista del progreso y el entendimiento del cuerpo con una sola perspectiva, desprovisto de la riqueza inter-transdisciplinaria.

A propósito del documento sobre el Plan Nacional de Recreación 1999-2002, se entiende el cuerpo de forma relevante desde un panorama que responde a la precarización de la salud, pues, tal como se menciona en el documento, las condiciones de salud biopsicosocial de la comunidad es un aspecto que influye sobre las políticas y la planificación en recreación. Según el concepto de recreación que subyace al plan, ésta debe contribuir al desarrollo armónico del hombre, y, por ende, a la salud del hombre y la mujer colombianos en todos sus aspectos. Lo anterior se argumenta a partir de cifras, las cuales demuestran que, en promedio, el $24 \%$ de la población, tanto de mujeres como de hombres, perciben su estado psicológico como preocupado, irritable, deprimido, angustiado. En cuanto al consumo de sustancias psicoactivas, el estudio acotado en el documento menciona que las bebidas alcohólicas se mantienen como la principal sustancia de inicio, con más del $60 \%$ de los casos en ambos sexos.

La crisis social actual, con innegables consecuencias sobre la percepción y la calidad de la salud de los colombianos, el ya sobrediagnosticado problema de violencia (intrafamiliar, política, callejera), la violación permanente de los derechos humanos (que, indudablemente, refleja una crisis de valores) y el consumo de sustancias lícitas e ilícitas, mencionados en el documento, con amplias cifras que lo respaldan, demandan a la recreación repensar sus conceptos y sus prácticas operacionalizando la intencionalidad de las actividades y estableciendo una clara 
relación entre los objetivos y los resultados esperados de sus actividades, así como documentando y socializando sus beneficios.

El Plan Nacional de Recreación enfoca su mirada en contribuir al fomento de una cultura de la salud, entendida como una actitud que se traduce en estilos de vida saludables en todas las dimensiones de la vida del hombre, y abordando la acción desde los factores estructurales que la afectan, y, por tanto, desde modelos integrales de prevención, promoción y educación donde la recreación ha encontrado una de sus mejores expresiones.

Adicionalmente a lo anterior, se presenta el documento sobre el Plan Nacional del Deporte, que plantea discusiones no únicamente referidas a la preparación atlética y a la actividad competitiva, sino que aduce al deporte como factor de comunicación social, embajador de la paz y elemento indispensable para el acercamiento y el entendimiento entre las personas (Aguilera, 1991).

No obstante, este plan asume nociones relacionadas con el deporte, en virtud de la buena salud combatiendo la nutrición descontrolada, el tabaquismo, algunos factores ecológicos (efectos de la contaminación medioambiental como ruido, rayos UV, calidad del aire, entre otros), las enfermedades cardiovasculares, el cáncer, la hipertensión, los trastornos psicológicos, las afecciones del aparato locomotor y otros, que agobian al hombre de hoy. Se entiende, entonces, el deporte como una herramienta para la mitigación de problemas de la salud, pero también para subsanar y mejorar el ambiente social, por cuanto es uno de los medios más efectivos para la lucha contra la narcomanía, la prostitución, el alcoholismo, la delincuencia y otras deformaciones sociales. Por todo esto, una de las direcciones prioritarias de la política social de cada país es la preocupación por la salud de sus habitantes, el desarrollo físico y su aptitud hacia el trabajo.

Así mismo, cabe mencionar la Ley 115 de 1994 (o Ley General de Educación), la cual tiene objetivos a partir de los cuales se intenta desarrollar una plena educación, y con tal fin propugna por el conocimiento del propio cuerpo de los infantes y de sus posibilidades de acción, así como por la adquisición de su identidad y su autonomía, como una forma de que las escuelas asuman la importancia de la corporalidad. De la misma forma, se ubican desde el artículo 21 de la citada ley objetivos específicos de la educación básica en el ciclo de primaria, normatividad en la cual especifica que los cinco primeros grados de la educación básica que constituyen el ciclo de primaria, deberán tener como propósitos particulares: la valoración de la higiene y la salud del propio cuerpo y la formación para la protección de la naturaleza y el ambiente; también, conocimiento y ejercitación del propio cuerpo, mediante la práctica de la educación física, la recreación y los deportes adecuados a su edad y conducentes a un desarrollo físico y armónico.

Al lado de esta normativa nacional, se encuentra la Ley 181 de 1995, a través de la cual se crean disposiciones para el fomento del deporte, la recreación, el aprovechamiento del tiempo libre y la educación física en el país. Particularmente, su entendido desde el amplio espectro del cuerpo concibe como educación física la disciplina científica cuyo objeto de estudio es la expresión corporal del hombre y la incidencia del movimiento en el desarrollo integral y en el mejoramiento de la salud y la calidad de vida de los individuos. Lo anterior denota claramente un arraigo conceptual que pone de relieve la condición física del individuo.

Ya en el plano internacional, son dignos de mención tres documentos de particular interés. Por un lado, la Carta Internacional de la Educación Física (1978), que concede una elevada importancia a la práctica de actividades que beneficien o comprometan la mejora de la salud y ayuden al desarrollo completo y armonioso del ser humano. De hecho, así lo menciona de manera específica el documento: "En el plano del individuo, la educación física y el deporte contribuyen a preservar y mejorar la salud, a proporcionar una sana ocupación del tiempo libre y a resistir mejor los inconvenientes de la vida moderna" (p. 2).

Por otro lado, el Manifiesto Mundial de la Educación Física (2000) incluye entre sus prioridades la práctica del ejercicio físico, a partir del cual se favorecerían un cuerpo sano y equilibrado, una aptitud para la acción y unos valores morales para convivir en paz. A raíz de la lectura del documento, se infiere un consenso entre todas las concepciones educativas de la educación física, el cual expresa que las actividades socio-psicomotrices se constituyen en un factor de equilibrio en la vida de las personas, hacia una plena interacción entre el espíritu y el cuerpo, la afectividad y la energía, el individuo y el grupo. 
Paralelamente, se menciona en el manifiesto que la educación física, como parte del proceso educativo, desenvuelve posibilidades de movimiento y educa para el entendimiento, pues es relevante y debe ser utilizada considerándola una experiencia única, por relacionarse con uno de los más preciosos recursos humanos, que es el cuerpo. De ahí que ayuda a los niños para que lleguen a una integración segura y a un adecuado desarrollo de la mente, el cuerpo y el equilibrio. Así mismo, es la única alternativa escolar cuyo foco principal es sobre el cuerpo, la actividad física, el desarrollo físico y la salud; contribuye, además, a que los niños desarrollen patrones de intereses en la actividad física, los cuales son esenciales para un desarrollo deseable y construyan los fundamentos para un estilo de vida saludable en la edad adulta.

Finalmente, el más actual de los documentos en el campo se titula: Educación Física de Calidad. Guía para Responsables Políticos. Se trata de una construcción colectiva abonada y auspiciada por la UNESCO (2015), y plantea necesidades contextuales para superar, como una posibilidad para el desarrollo humano y social de los países partiendo de la implementación debida de la educación física. Así mismo, aduce que la educación física, como única asignatura curricular cuyo enfoque combina la competencia corporal y física con la comunicación y el aprendizaje basado en valores, representa un portal de aprendizaje para desarrollar las aptitudes necesarias para tener éxito en el siglo XXI.

Las acepciones sobre el cuerpo, tal como lo indica el documento, pasan por el entendido de la actividad física y la salud como una alternativa para el desarrollo de experiencias de aprendizaje en niños y jóvenes. Su corporalidad se ve potenciada en la medida de las vivencias, las habilidades psicomotrices, la comprensión cognitiva y las aptitudes sociales y emocionales requeridas para una vida sana y físicamente activa.

\section{BREVE DISCUSIÓN}

A partir de cada una de las políticas, los documentos, las leyes y las propuestas de orden normativo arriba referenciados, se ha brindado una panorámica sobre la educación física y sus conceptos y nociones en torno al cuerpo, independientemente de su naturaleza discursiva o de sus intereses educativos, académicos o gerenciales. Algunos textos, como se ha notado, guardan relación en mayor medida con un cuerpo que se vincula a lo simbólico, lo humano, lo social y lo cultural (lineamientos curriculares, orientaciones pedagógicas, plan nacional de educación física, educación física de calidad, entre otras), contrariamente a otras que establecen afinidades con discursos propios de la actividad física y el ejercicio físico en procura del mejoramiento de la salud y la condición física (plan nacional de recreación, plan nacional de deporte, entre otras). No obstante, el interés en proporcionar una descripción sobre el cuerpo en materia de normativas en los planos nacional e internacional facilita el reconocimiento y el auscultamiento a la hora de levantar el archivo objeto de análisis.

Lo anterior se comprende en el espectro de una parcial discusión, debido a que, por un lado, la revisión archivística se consume en la hojeada documental, tal como aquí se presentó, respecto a las prácticas discursivas que sobre el cuerpo se encuentran inmersas y son apropiadas por el programa de Licenciatura en Educación Física y Deportes de la Universidad de los Llanos; y, por otro, porque resta el desarrollo de entrevistas en profundidad que permitan interrogar el trasegar discursivo del cuerpo, lo que, sin duda, hará del estudio un complejo escenario de análisis e interpretación sobre la historia (irreductible) corporal de esta casa de altos estudios.

\section{REFERENCIAS}

Aguilera, R. (1991). El deporte de hoy: realidades y perspectivas. La Habana: Editorial Científico Técnica.

Carta Internacional de la Educación Física. UNESCO. 1978. En: http://portal.unesco.org/es/ev.php-URL_ ID $=13150 \&$ URL_DO=DO_TOPIC\&URL_SECTION $=201$. html.

Educación Física de Calidad: Guía para Responsables Políticos. (2015). UNESCO. En: http://unesdoc. unesco.org/images/0023/002313/231340s.pdf.

Fideli, R. \& Marradi, A. (1996). Entrevista. Enciclopedia delle Scienze Sociali, V, 71-82. Roma: Instituto Della Enciclopedia Italiana.

Latorre, A., Rincón, D. \& Arnal, J. (2003). Bases metodológicas de la investigación educativa. Barcelona: Experiencia.

Ley 115 de 1994. Ministerio de Educación Nacional. Bogotá. En: http://www.mineducacion.gov.co/1621/ article-85906.html. 
Ley 181 de 1995. Ministerio de Educación Nacional. Bogotá. En: http://www.mineducacion.gov.co/1621/ articles-85919_archivo_pdf.pdf.

Lineamientos Curriculares de la Educación Física (1996). Ministerio de Educación Nacional. Bogotá. En: http:// www.mineducacion.gov.co/1621/articles-89869_ archivo_pdf3.pdf.

Manifiesto Mundial de la Educación Física (2000). Federación Internacional de Educación Física. En: http://www.fiepsurdelmundo.cl/que-es-fiep/.

Mockus, A. (1992). Educación física y pedagogía latinoamericana. Santafé de Bogotá.

Mauss, M. (1979). Sociología y Antropología. Madrid: Tecnos.

Orientaciones Pedagógicas paralaEducación Física (2010). Ministerio de Educación Nacional. Bogotá. En: http:// www.mineducacion.gov.co/1759/articles-340033_ archivo_pdf_Orientaciones_EduFisica_Rec_Deporte. pdf.
Pedraza, S. (1998). La cultura somática de la modernidad. Cultura, política y Modernidad. Santafé de Bogotá: Ces. Universidad Nacional.

Plan Nacional de Educación Física (2002). Fundación Colombiana de Tiempo Libre y Recreación. Bogotá. En: http://www.funlibre.org/biblioteca/items/show/ 511.

Plan Nacional de Recreación (1999). Fundación Colombiana de Tiempo Libre y Recreación. Bogotá. En: http://www.redcreacion.org/documentos/planna cionalrecreacion/prinicipal.htm.

Plan Nacional del Deporte (2001). COLDEPORTES. Bogotá. En: http://siscred.scrd.gov.co/biblioteca/bitstream/ 123456789/38/1/Plan\%20nacional\%20deporte.pdf. 